\title{
Monotone Solution of Cauchy Type Weighted Nonlocal Fractional Differential Equation
}

\author{
Mohammed Mazhar UI Haque ${ }^{1, a *}$, Bhausaheb R. Sontakke ${ }^{2, b}$, \\ Tarachand L. Holambe ${ }^{3, c}$ \\ ${ }^{1}$ Department of Mathematics, Dr. B.A.M. University, Aurangabad, Maharashtra, India \\ ${ }^{2}$ Department of Mathematics, Pratishthan College, India \\ ${ }^{3}$ Department of Mathematics, Kai Shankarrao Gutte ACS College, Dharmapuri, Beed, Maharashtra, \\ India \\ amazhar-ul-haque@hotmail.com, \\ bbrsontakke@rediffmail.com, ctarachandholambe@gmail.com
}

Keywords: fractional differential and integral equation; Nonlocal problems; approximate solution.

\begin{abstract}
In this paper we will consider a nonlinear fractional differential equation with weighted initial and nonlocal conditions and will obtain monotone solution by the sequence of successive approximations starting at a lower solution converges monotonically to the solution of the related Cauchy type weighted nonlocal fractional differential equation under some suitable conditions.
\end{abstract}

MSC2010: 5G10, 47H09, 47H10.

\section{Introduction}

Fractional derivatives now used to model a wide variety of real life problems and it has applications in physics, finance, biology, hydrology, etc., as fractional order models can be found to be more adequate than integer order models in some real world problems because fractional derivatives provide an excellent tool for the description of memory and hereditary properties of various materials and processes.

Nonlinear fractional differential equation with weighted initial data has been studied by several authors. The weighted Cauchy-type problem

$$
\begin{array}{r}
D^{\alpha}(u(t))=f(t, u(t)) \\
\left.t^{1-\alpha} u(t)\right|_{t=0}=b
\end{array}
$$

studied by Khaled et al. [13].

The solution of the periodic boundary value problem for a fractional differential equation involving a Riemann-Liouville fractional derivative

$$
\begin{array}{r}
D^{\alpha}(u(t))=f(t, u(t)) \\
\left.t^{1-\alpha} u(t)\right|_{t=0}=\left.t^{1-\alpha} u(t)\right|_{t=T}
\end{array}
$$

studied by Weia et al. [14]. Also the existence of solutions of fractional equations of Volterra type with the Riemann-Liouville derivative,

$$
\begin{array}{r}
D^{\alpha}(u(t))=f\left(t, u(t), \int_{0}^{t} k(t, s) u(s) d s\right) \\
\left.t^{1-\alpha} u(t)\right|_{t=0}=r
\end{array}
$$

studied by Jankowski [15] etc. And the weighted non-local fractional differential equation 


$$
\begin{array}{r}
{ }^{c} D^{\alpha}(u(t))=f(t, u(t)) \\
\lim _{t \rightarrow 0^{+}} t^{1-\alpha} u(t)=\sum_{i=1}^{m} a_{i} u\left(\tau_{i}\right)
\end{array}
$$

studied by Holambe et al. [26, 27, 28] and the references therein. Problems in nonlinear fractional differential equation were studied by various researchers.

The importance of non-local problems appears to have been first noted in the literature by Bitsadze Samarski [12]. By Byszewski [1,2], the non-local condition can be more useful than the standard initial condition to describe some physical phenomena.

Now here we consider the weighted non-local fractional differential equation

$$
\begin{aligned}
& { }^{c} D^{\alpha}\left(\frac{p(t) u(t)}{g(t, u(t))}\right)=f(t, u(t)) \\
& \lim _{t \rightarrow 0^{+}} t^{1-\alpha} p(t) u(t)=\sum_{i=1}^{m} a_{i} u\left(\tau_{i}\right)
\end{aligned}
$$

where ${ }^{c} D^{\alpha}$ is Caputo fractional derivatives of order $0<\alpha \leq 1$ and $0<t \leq T<\infty, 0<\tau_{i}<T<\infty$.

\section{Auxiliary Results}

We need certain definitions,lemmas and theorems in the sequel.

We will use extensively studied fractional derivative and integral with its properties by $[6,7,10$, $11,16,17,18,19,20,23,24,25]$

Let $E$ denote a partially ordered real normed linear space with an order relation $\preceq$ and the norm $\|\cdot\|$. It is known that $E$ is regular if $\left\{x_{n}\right\}_{n \in \mathbb{N}}$ is a non-decreasing (resp. non-increasing) sequence in $E$ such that $x_{n} \rightarrow x^{*}$ as $n \rightarrow \infty$, then $x_{n} \preceq x^{*}\left(\right.$ resp. $x_{n} \succeq x^{*}$ ) for all $n \in \mathbb{N}$. Clearly, the partially ordered Banach space $C(J, \mathbb{R})$ is regular and the conditions guaranteeing the regularity of any partially ordered normed linear space $E$ may be found in Heikkilä and Lakshmikantham [8] and the references therein.

Definition 1. A mapping $\mathcal{T}: E \rightarrow E$ is called isotone or non-decreasing if it preserves the order relation $\preceq$, that is, if $x \preceq y$ implies $\mathcal{T} x \preceq \mathcal{T} y$ for all $x, y \in E$.

Definition 2 [3]. A mapping $\mathcal{T}: E \rightarrow E$ is called partially continuous at a point $a \in E$ if for $\epsilon>0$ there exists a $\delta>0$ such that $\|\mathcal{T} x-\mathcal{T} a\|<\epsilon$ whenever $x$ is comparable to $a$ and $\|x-a\|<\delta . \mathcal{T}$ called partially continuous on $E$ if it is partially continuous at every point of it. It is clear that if $\mathcal{T}$ is partially continuous on $E$, then it is continuous on every chain $C$ contained in $E$.

Definition 3. A mapping $\mathcal{T}: E \rightarrow E$ is called partially bounded if $\mathcal{T}(C)$ is bounded for every chain $C$ in $E . \mathcal{T}$ is called uniformly partially bounded if all chains $\mathcal{T}(C)$ in $E$ are bounded by a unique constant. $\mathcal{T}$ is called bounded if $\mathcal{T}(E)$ is a bounded subset of $E$.

Definition 4. A mapping $\mathcal{T}: E \rightarrow E$ is called partially compact if $\mathcal{T}(C)$ is a relatively compact subset of $E$ for all totally ordered sets or chains $C$ in $E$. $\mathcal{T}$ is called uniformly partially compact if $\mathcal{T}(C)$ is a uniformly partially bounded and partially compact on $E$. $\mathcal{T}$ is called partially totally bounded if for any totally ordered and bounded subset $C$ of $E, \mathcal{T}(C)$ is a relatively compact subset of $E$. If $\mathcal{T}$ is partially continuous and partially totally bounded, then it is called partially completely continuous on $E$. 
Definition 5 [3]. The order relation $\preceq$ and the metric $d$ on a non-empty set $E$ are said to be compatible if $\left\{x_{n}\right\}_{n \in \mathbb{N}}$ is a monotone, that is, monotone nondecreasing or monotone nonincreasing sequence in $E$ and if a subsequence $\left\{x_{n_{k}}\right\}_{n \in \mathbb{N}}$ of $\left\{x_{n}\right\}_{n \in \mathbb{N}}$ converges to $x^{*}$ implies that the original sequence $\left\{x_{n}\right\}_{n \in \mathbb{N}}$ converges to $x^{*}$. Similarly, given a partially ordered normed linear space $(E, \preceq,\|\cdot\|)$, the order relation $\preceq$ and the norm $\|\cdot\|$ are said to be compatible if $\preceq$ and the metric $d$ defined through the norm $\|\cdot\|$ are compatible.

Definition 6 [21]. An upper semi-continuous and monotone nondecreasing function $\psi: \mathbb{R}_{+} \rightarrow \mathbb{R}_{+}$ is called a $\mathcal{D}$-function provided $\psi(r)=0$ iff $r=0$. Let $(E, \preceq,\|\cdot\|)$ be a partially ordered normed linear space. A mapping $\mathcal{T}: E \rightarrow E$ is called partially nonlinear $\mathcal{D}$-Lipschitz if there exists a $\mathcal{D}$-function $\psi: \mathbb{R}_{+} \rightarrow \mathbb{R}_{+}$such that

$$
\|\mathcal{T} x-\mathcal{T} y\| \leq \psi(\|x-y\|)
$$

for all comparable elements $x, y \in E$. If $\psi(r)=k r, k>0$, then $\mathcal{T}$ is called a partially Lipschitz with a Lipschitz constant $k$.

Let $(E, \preceq,\|\cdot\|)$ be a partially ordered normed linear algebra. Denote

$$
E^{+}=\{x \in E \mid x \succeq \theta, \text { where } \theta \text { is the zero element of } E\}
$$

and

$$
\mathcal{K}=\left\{E^{+} \subset E \mid u v \in E^{+} \text {for all } u, v \in E^{+}\right\} .
$$

The elements of $\mathcal{K}$ are called the positive vectors of the normed linear algebra $E$. The following lemma follows immediately from the definition of the set $\mathcal{K}$ and which is often times used in the applications of hybrid fixed point theory in Banach algebras.

Lemma 7 [22]. If $u_{1}, u_{2}, v_{1}, v_{2} \in \mathcal{K}$ are such that $u_{1} \preceq v_{1}$ and $u_{2} \preceq v_{2}$, then $u_{1} u_{2} \preceq v_{1} v_{2}$.

Definition 8. An operator $\mathcal{T}: E \rightarrow E$ is said to be positive if the range $R(\mathcal{T})$ of $\mathcal{T}$ is such that $R(\mathcal{T}) \subseteq \mathcal{K}$

The method may be stated as "the monotonic convergence of the sequence of successive approximations to the solutions of a nonlinear equation beginning with a lower or an upper solution of the equation as its initial or first approximation" which is a powerful tool in the existence theory of nonlinear analysis. It is clear that the method is different from the usual Picard's successive iteration method and embodied in the following applicable hybrid fixed point theorems proved in[3] which forms a useful key tool for our work contained in this paper. A few other hybrid fixed point theorems involving the method may be found in $[3,4,5]$.

Theorem 9 [4]. Let $(E, \preceq,\|\cdot\|)$ be a regular partially ordered complete normed linear algebra such that the order relation $\preceq$ and the norm $\|\cdot\|$ in $E$ are compatible in every compact chain of E. Let $\mathcal{A}, \mathcal{B}: E \rightarrow \mathcal{K}$ be two nondecreasing operators such that

(a) $\mathcal{A}$ is partially bounded and partially nonlinear $\mathcal{D}$-Lipschitz with $\mathcal{D}$-functions $\psi_{\mathcal{A}}$,

(b) $\mathcal{B}$ is partially continuous and uniformly partially compact, and

(c) $M \psi_{\mathcal{A}}(r)<r, r>0$, where $M=\sup \{\|\mathcal{B}(C)\|: C$ is a chain in $E\}$, and

(d) there exists an element $x_{0} \in X$ such that $x_{0} \preceq \mathcal{A} x_{0} \mathcal{B} x_{0}$ or $x_{0} \succeq \mathcal{A} x_{0} \mathcal{B} x_{0}$. 
Then the operator equation

$$
\mathcal{A} x \mathcal{B} x=x
$$

has a solution $x^{*}$ in $E$ and the sequence $\left\{x_{n}\right\}$ of successive iterations defined by $x_{n+1}=\mathcal{A} x_{n} \mathcal{B} x_{n}$, $n=0,1, \ldots$, converges monotonically to $x^{*}$.

Remark. The compatibility of the order relation $\preceq$ and the norm $\|\cdot\|$ in every compact chain of $E$ holds if every partially compact subset of $E$ possesses the compatibility property with respect to $\preceq$ and $\|\cdot\|$. Note that a subset $S$ of the partially ordered Banach space $C(J, \mathbb{R})$ is called partially compact if every chain $C$ in $S$ is compact. This simple fact has been utilized to prove the main results of this paper.

\section{Main Results}

The equaivalent integral form of the problem [5] is considered in the function space $C(J, \mathbb{R})$ of continuous real-valued functions defined on $J$. We define a norm $\|\cdot\|$ and the order relation $\leq$ in $C(J, \mathbb{R})$ by

$$
\|x\|=\sup _{t \in J}|x(t)|
$$

and

$$
x \leq y \Longleftrightarrow x(t) \leq y(t)
$$

for all $t \in J$ respectively. Clearly, $C(J, \mathbb{R})$ is a Banach algebra with respect to above supremum norm and is also partially ordered w.r.t. the above partially order relation $\leq$. It is known that the partially ordered Banach algebra $C(J, \mathbb{R})$ has some nice properties concerning the compatibility property with respect to the norm $\|\cdot\|$ and the order relation $\leq$ in certain subsets of it. The following lemma in this connection follows by an application of Arzelá-Ascoli theorem.

Lemma 10. Let $(C(J, \mathbb{R}), \leq,\|\cdot\|)$ be a partially ordered Banach space with the norm $\|\cdot\|$ and the order relation $\leq$ defined by (9) and (10) respectively. Then $\|\cdot\|$ and $\leq$ are compatible in every partially compact subset of $C(J, \mathbb{R})$.

Proof. The lemma mentioned in Dhage [4], but the proof appears in Dhage [5].

We need the following definition in what follows.

Definition 11. A function $u_{l} \in C(J, \mathbb{R})$ is said to be a lower solution of the problem (5) if it satisfies

$$
\begin{aligned}
{ }^{c} D^{\alpha}\left(\frac{p(t) u_{l}(t)}{g\left(t, u_{l}(t)\right)}\right) & \leq f\left(t, u_{l}(t)\right) \\
\lim _{t \rightarrow 0^{+}} t^{1-\alpha} p(t) u_{l}(t) & \leq \sum_{i=1}^{m} a_{i} u_{l}\left(\tau_{i}\right)
\end{aligned}
$$

for all $t \in J$. Similarly, a function $u_{u} \in C(J, \mathbb{R})$ is said to be an upper solution of the problem (5) if it satisfies the above inequalities with reverse sign.

Definition 12. A function $f(t, u)$ is called Carathéodory if

(i) the map $t \mapsto f(t, u)$ is measurable for each $u \in \mathbb{R}$ and

(ii) the map $u \mapsto f(t, u)$ is continuous for each $t \in J$. 
A Caratheódory function $f$ is called $L^{2}$-Carathéodory if

(iii) there exists a function $h \in L^{2}(J, \mathbb{R})$ such that

$$
|f(t, u)| \leq h(t) \text { a.e. } t \in J .
$$

We consider the following set of assumptions in what follows:

$\left(\mathrm{A}_{1}\right)$ The functions $f, g: J \times \mathbb{R} \rightarrow \mathbb{R}_{+}, \alpha: J \rightarrow \mathbb{R}_{+}$where $\alpha$ is continuous function.

$\left(\mathrm{A}_{2}\right)$ There exist constants $M, M_{f}, M_{g}>0$ such that $0 \leq t^{\alpha-1} \leq M, 0 \leq g(t, x) \leq M_{g}$ and $0 \leq f(t, x) \leq M_{f}$ for all $t \in J$ and $x \in \mathbb{R}$.

$\left(\mathrm{A}_{3}\right)$ There exists a $\mathcal{D}$-function $\psi_{f}$ such that

$$
0 \leq g(t, x)-g(t, y) \leq \psi_{f}(x-y)
$$

for all $t \in J$ and $x, y \in \mathbb{R}, x \leq y$.

( $\left.\mathrm{A}_{4}\right) f(t, x)$ is nondecreasing in $x$ for all $t \in J$.

$\left(\mathrm{A}_{5}\right)$ The problem (5) has a lower solution $u_{l} \in C(J, \mathbb{R})$.

The following lemma is useful in what follows.

Lemma 13. For any $f \in C(J \times \mathbb{R}, \mathbb{R}))$, if $u$ is a solution of the problem

$$
\begin{gathered}
{ }^{c} D^{\alpha}\left(\frac{p(t) u(t)}{g(t, u(t))}\right)=f(t, u(t)) \\
\lim _{t \rightarrow 0^{+}} t^{1-\alpha} p(t) u(t)=\sum_{i=1}^{m} a_{i} u\left(\tau_{i}\right) \\
0<\alpha \leq 1, \quad 0<t, \tau_{i}<T<\infty
\end{gathered}
$$

then

$$
\begin{gathered}
u(t)=g(t, u(t))\left\{\frac{K t^{\alpha-1}}{p(t)} \sum_{i=1}^{m} a_{i} \frac{g\left(\tau_{i}, u\left(\tau_{i}\right)\right.}{p\left(\tau_{i}\right) \Gamma(\alpha)} \int_{0}^{\tau_{i}}\left(\tau_{i}-s\right)^{\alpha-1} f(s, u(s)) d s\right. \\
\left.+\frac{1}{p(t) \Gamma(\alpha)} \int_{0}^{t}(t-s)^{\alpha-1} f(s, u(s)) d s\right\}
\end{gathered}
$$

where $K=\left(k_{0}-\sum_{i=1}^{m} \frac{a_{i} g\left(\tau_{i}, u\left(\tau_{i}\right)\right.}{\tau_{i}^{1-\alpha} p\left(\tau_{i}\right)}\right)^{-1}$ as $\lim _{t \rightarrow 0+} g(t, u(t))=k_{0} \neq 0$ and vice-versa.

Proof. For the nonlocal weighted fractional differential equation (5) by the Caputo fractional derivative of order $\gamma>0$

$$
{ }^{c} D_{a}^{\alpha} f(t)=\frac{1}{\Gamma(n-\alpha)} \int_{a}^{t}(t-s)^{n-\alpha-1} f^{n}(s) d s, n-1<\alpha \leq n, n \in \mathbb{N}
$$

for $n=1, a=0$

$$
{ }^{c} D^{\alpha} f(t)=\frac{1}{\Gamma(1-\alpha)} \int_{0}^{t}(t-s)^{-\alpha} f^{1}(s) d s
$$


By the definition of Reimann-Liouville fractional integral

$$
{ }^{c} D^{\alpha} f(t)=\frac{d}{d t} I^{1-\alpha} f(t)
$$

from (5)

$$
{ }^{c} D^{\alpha}\left(\frac{p(t) u(t)}{g(t, u(t))}\right)=\frac{d}{d t} I^{1-\alpha}\left(\frac{p(t) u(t)}{g(t, u(t))}\right)=f(t, u(t)) .
$$

Integrating from 0 to $t$,we get

$$
\begin{aligned}
I^{1-\alpha}\left(\frac{p(t) u(t)}{g(t, u(t))}\right)-\left.I^{1-\alpha}\left(\frac{p(t) u(t)}{g(t, u(t))}\right)\right|_{t=0} & =\int_{0}^{t} f(s, u(s)) d s \\
I^{1-\alpha}\left(\frac{p(t) u(t)}{g(t, u(t))}\right)-C & =\int_{0}^{t} f(s, u(s)) d s .
\end{aligned}
$$

Operating Reimann-Liouville integral $I^{\alpha}$ on bothside

$$
\begin{aligned}
& I^{\alpha} I^{1-\alpha}\left(\frac{p(t) u(t)}{g(t, u(t))}\right)-I^{\alpha} C=I^{\alpha+1} f(t, u(t)) \\
& I\left(\frac{p(t) u(t)}{g(t, u(t))}\right)-\frac{C t^{\alpha}}{\Gamma(\alpha+1)}=I^{\alpha+1} f(t, u(t)) .
\end{aligned}
$$

Taking differentiation on both side

$$
\begin{array}{r}
\left(\frac{p(t) u(t)}{g(t, u(t))}\right)-\frac{C t^{\alpha-1}}{\Gamma(\alpha)}=\frac{1}{\Gamma(\alpha)} \int_{0}^{t}(t-s)^{\alpha-1} f(s, u(s)) d s \\
t^{1-\alpha}\left(\frac{p(t) u(t)}{g(t, u(t))}\right)=\frac{C}{\Gamma(\alpha)}+\frac{1}{\Gamma(\alpha)} t^{1-\alpha} \int_{0}^{t}(t-s)^{\alpha-1} f(s, u(s)) d s
\end{array}
$$

now

$$
\lim _{t \rightarrow 0+} t^{1-\alpha}\left(\frac{p(t) u(t)}{g(t, u(t))}\right)=\frac{C}{\Gamma(\alpha)}
$$

since $\lim _{t \rightarrow 0+} g(t, u(t))=k_{0} \neq 0$ from (5)

$$
\begin{array}{r}
\lim _{t \rightarrow 0^{+}} t^{1-\alpha} p(t) u(t)=\sum_{i=1}^{m} a_{i} u\left(\tau_{i}\right) \\
\frac{C}{\Gamma(\alpha)}=\frac{1}{k_{0}} \sum_{i=1}^{m} a_{i} u\left(\tau_{i}\right)
\end{array}
$$

put $t=\tau_{i}$ in the equation (11) 


$$
\begin{array}{r}
\tau_{i}^{1-\alpha}\left(\frac{p\left(\tau_{i}\right) u\left(\tau_{i}\right)}{g\left(\tau_{i}, u\left(\tau_{i}\right)\right)}\right)=\frac{C}{\Gamma(\alpha)}+\frac{1}{\Gamma(\alpha)} \tau_{i}^{1-\alpha} \int_{0}^{\tau_{i}}\left(\tau_{i}-s\right)^{\alpha-1} f(s, u(s)) d s \\
\left(\frac{u\left(\tau_{i}\right)}{g\left(\tau_{i}, u\left(\tau_{i}\right)\right)}\right)=\frac{C}{\tau_{i}^{1-\alpha} p\left(\tau_{i}\right) \Gamma(\alpha)}+\frac{1}{p\left(\tau_{i}\right) \Gamma(\alpha)} \int_{0}^{\tau_{i}}\left(\tau_{i}-s\right)^{\alpha-1} f(s, u(s)) d s \\
u\left(\tau_{i}\right)=g\left(\tau_{i}, u\left(\tau_{i}\right)\right)\left\{\frac{C}{\tau_{i}^{1-\alpha} p\left(\tau_{i}\right) \Gamma(\alpha)}+\frac{1}{p\left(\tau_{i}\right) \Gamma(\alpha)} \int_{0}^{\tau_{i}}\left(\tau_{i}-s\right)^{\alpha-1} f(s, u(s)) d s\right\} \\
\sum_{i=1}^{m} a_{i} u\left(\tau_{i}\right)=\sum_{i=1}^{m} \frac{g\left(\tau_{i}, u\left(\tau_{i}\right)\right) a_{i} C}{\tau_{i}^{1-\alpha} p\left(\tau_{i}\right) \Gamma(\alpha)}+\sum_{i=1}^{m} \frac{a_{i} g\left(\tau_{i}, u\left(\tau_{i}\right)\right)}{p\left(\tau_{i}\right) \Gamma(\alpha)} \int_{0}^{\tau_{i}}\left(\tau_{i}-s\right)^{\alpha-1} f(s, u(s)) d s \\
\frac{k_{0} C}{\Gamma(\alpha)}=\sum_{i=1}^{m} \frac{g\left(\tau_{i}, u\left(\tau_{i}\right)\right) a_{i} C}{\tau_{i}^{1-\alpha} p\left(\tau_{i}\right) \Gamma(\alpha)}+\sum_{i=1}^{m} \frac{a_{i} g\left(\tau_{i}, u\left(\tau_{i}\right)\right)}{p\left(\tau_{i}\right) \Gamma(\alpha)} \int_{0}^{\tau_{i}}\left(\tau_{i}-s\right)^{\alpha-1} f(s, u(s)) d s \\
\left(k_{0}-\sum_{i=1}^{m} \frac{a_{i} g\left(\tau_{i}, u\left(\tau_{i}\right)\right.}{\tau_{i}^{1-\alpha} p\left(\tau_{i}\right)}\right) \frac{C}{\Gamma(\alpha)}=\sum_{i=1}^{m} \frac{a_{i} \frac{g\left(\tau_{i}, u\left(\tau_{i}\right)\right.}{p\left(\tau_{i}\right) \Gamma(\alpha)} \int_{0}^{\tau_{i}}\left(\tau_{i}-s\right)^{\alpha-1} f(s, u(s)) d s}{\frac{m}{\tau_{i}}}=K \sum_{i=1}^{m} \frac{g\left(\tau_{i}, u\left(\tau_{i}\right)\right.}{p\left(\tau_{i}\right) \Gamma(\alpha)} \int_{0}^{\tau_{i}}\left(\tau_{i}-s\right)^{\alpha-1} f(s, u(s)) d s
\end{array}
$$

where $K=\left(k_{0}-\sum_{i=1}^{m} \frac{a_{i} g\left(\tau_{i}, u\left(\tau_{i}\right)\right.}{\tau_{i}^{1-\alpha} p\left(\tau_{i}\right)}\right)^{-1}$. Thus from (11)

$$
\begin{aligned}
\left(\frac{u(t)}{g(t, u(t))}\right)= & \frac{K t^{\alpha-1}}{p(t)} \sum_{i=1}^{m} a_{i} \frac{g\left(\tau_{i}, u\left(\tau_{i}\right)\right.}{p\left(\tau_{i}\right) \Gamma(\alpha)} \int_{0}^{\tau_{i}}\left(\tau_{i}-s\right)^{\alpha-1} f(s, u(s)) d s \\
& +\frac{1}{p(t) \Gamma(\alpha)} \int_{0}^{t}(t-s)^{\alpha-1} f(s, u(s)) d s \\
u(t)= & g(t, u(t))\left\{\frac{K t^{\alpha-1}}{p(t)} \sum_{i=1}^{m} a_{i} \frac{g\left(\tau_{i}, u\left(\tau_{i}\right)\right.}{p\left(\tau_{i}\right) \Gamma(\alpha)} \int_{0}^{\tau_{i}}\left(\tau_{i}-s\right)^{\alpha-1} f(s, u(s)) d s\right. \\
& \left.+\frac{1}{p(t) \Gamma(\alpha)} \int_{0}^{t}(t-s)^{\alpha-1} f(s, u(s)) d s\right\}
\end{aligned}
$$

Theorem 14. Assume that hypotheses $\left(A_{1}\right)-\left(A_{5}\right)$ hold. Furthermore, assume that

$$
\left(\frac{K M\left[M_{G}+1\right] T^{\alpha-1} M_{f}}{p(T) \Gamma(\alpha)}\right) \psi_{f}(r)<r, r>0
$$

then the FDE(5) has a solution $x^{*}$ defined on $J$ and the sequence $\left\{x_{n}\right\}_{n \in \mathbb{N} \cup\{0\}}$ of successive approximations defined by 


$$
\begin{aligned}
x_{n+1}(t)=g\left(t, x_{n}(t)\right)\left\{\frac{K t^{\alpha-1}}{p(t)} \sum_{i=1}^{m} a_{i} \frac{g\left(\tau_{i}, x_{n}\left(\tau_{i}\right)\right.}{p\left(\tau_{i}\right) \Gamma(\alpha)} \int_{0}^{\tau_{i}}\left(\tau_{i}-s\right)^{\alpha-1} f\left(s, x_{n}(s)\right) d s\right. \\
\left.+\frac{1}{p(t) \Gamma(\alpha)} \int_{0}^{t}(t-s)^{\alpha-1} f\left(s, x_{n}(s)\right) d s\right\}
\end{aligned}
$$

for all $t \in J$, where $x_{0}=a$, converges monotonically to $x^{*}$.

Proof. Set $E=C(J, \mathbb{R})$. Then, from Lemma 10 it follows that every compact chain in $E$ possesses the compatibility property with respect to the norm $\|\cdot\|$ and the order relation $\leq$ in $E$.

Define two operators $\mathcal{A}$ and $\mathcal{B}$ on $E$ by

$$
\begin{gathered}
\mathcal{A} x(t)=g(t, x(t)), t \in J, \\
\mathcal{B} x(t)=\left\{\frac{K t^{\alpha-1}}{p(t)} \sum_{i=1}^{m} a_{i} \frac{g\left(\tau_{i}, x\left(\tau_{i}\right)\right.}{p\left(\tau_{i}\right) \Gamma(\alpha)} \int_{0}^{\tau_{i}}\left(\tau_{i}-s\right)^{\alpha-1} f(s, x(s)) d s\right. \\
\left.+\frac{1}{p(t) \Gamma(\alpha)} \int_{0}^{t}(t-s)^{\alpha-1} f(s, x(s)) d s\right\}, t \in J .
\end{gathered}
$$

From the continuity of the integral and the hypotheses $\left(\mathrm{A}_{1}\right)-\left(\mathrm{A}_{5}\right)$, it follows that $\mathcal{A}$ and $\mathcal{B}$ define the maps $\mathcal{A}, \mathcal{B}: E \rightarrow \mathcal{K}$. Now by definitions of the operators $\mathcal{A}$ and $\mathcal{B}$, the $\operatorname{FDE}(5)$ is equivalent to the operator equation

$$
\mathcal{A} x(t) \mathcal{B} x(t)=x(t), \quad t \in J
$$

We shall show that the operators $\mathcal{A}, \mathcal{B}$ satisfy all the conditions of Theorem 9 . This is achieved in the series of following steps.

Step I: $\mathcal{A}, \mathcal{B}$ are nondecreasing on $E$.

Let $x, y \in E$ be such that $x \geq y$. Then by hypothesis $\left(\mathrm{A}_{2}\right),\left(\mathrm{A}_{3}\right)$ and $\left(\mathrm{A}_{4}\right)$, we obtain

$$
\mathcal{A} x(t)=g(t, x(t)) \geq g(t, y(t))=\mathcal{A} y(t),
$$

and

$$
\begin{aligned}
\mathcal{B} x(t)= & \left\{\frac{K t^{\alpha-1}}{p(t)} \sum_{i=1}^{m} a_{i} \frac{g\left(\tau_{i}, x\left(\tau_{i}\right)\right.}{p\left(\tau_{i}\right) \Gamma(\alpha)} \int_{0}^{\tau_{i}}\left(\tau_{i}-s\right)^{\alpha-1} f(s, x(s)) d s\right. \\
& \left.+\frac{1}{p(t) \Gamma(\alpha)} \int_{0}^{t}(t-s)^{\alpha-1} f(s, x(s)) d s\right\} \\
\geq & \left\{\frac{K t^{\alpha-1}}{p(t)} \sum_{i=1}^{m} a_{i} \frac{g\left(\tau_{i}, y\left(\tau_{i}\right)\right.}{p\left(\tau_{i}\right) \Gamma(\alpha)} \int_{0}^{\tau_{i}}\left(\tau_{i}-s\right)^{\alpha-1} f(s, y(s)) d s\right. \\
& \left.+\frac{1}{p(t) \Gamma(\alpha)} \int_{0}^{t}(t-s)^{\alpha-1} f(s, y(s)) d s\right\}=\mathcal{B} y(t)
\end{aligned}
$$

for all $t \in J$. This shows that $\mathcal{A}$ and $\mathcal{B}$ are nondecreasing operators on $E$ into $E$. Thus, $\mathcal{A}, \mathcal{B}$ are nondecreasing positive operators on $E$ into itself. 
Step II: $\mathcal{A}$ is partially bounded and partially $\mathcal{D}$-Lipschitz on $E$.

Let $x \in E$ be arbitrary. Then by $\left(\mathrm{A}_{2}\right)$,

$$
|\mathcal{A} x(t)| \leq \mid g(t, x(t))) \mid \leq M_{g},
$$

for all $t \in J$. Taking supremum over $t$, we obtain $\|\mathcal{A} x\| \leq M_{g}$ and so, $\mathcal{A}$ is bounded. This further implies that $\mathcal{A}$ is partially bounded on $E$.

Next, let $x, y \in E$ be such that $x \leq y$. Then, by hypothesis $\left(\mathrm{A}_{3}\right)$,

$$
|\mathcal{A} x(t)-\mathcal{A} y(t)|=|g(t, x(t))-g(t, y(t))| \leq \psi_{g}|x(t)-y(t)| \leq \psi_{g}(\|x-y\|),
$$

for all $t \in J$. Taking supremum over $t$, we obtain

$$
\|\mathcal{A} x-\mathcal{A} y\| \leq \psi_{g}(\|x-y\|)
$$

for all $x, y \in E$ with $x \leq y$. Hence $\mathcal{A}$ is partially nonlinear $\mathcal{D}$-Lipschitz operators on $E$ which further implies that they are also a partially continuous on $E$ into itself.

Step III: $\mathcal{B}$ is a partially continuous operator on $E$.

Let $\left\{x_{n}\right\}_{n \in \mathbb{N}}$ be a sequence in a chain $C$ of $E$ such that $x_{n} \rightarrow x$ for all $n \in \mathbb{N}$. Then, by dominated convergence theorem, we have

$$
\begin{gathered}
\lim _{n \rightarrow \infty} \mathcal{B} x_{n}(t)=\lim _{n \rightarrow \infty}\left\{\frac{K t^{\alpha-1}}{p(t)} \sum_{i=1}^{m} a_{i} \frac{g\left(\tau_{i}, x_{n}\left(\tau_{i}\right)\right.}{p\left(\tau_{i}\right) \Gamma(\alpha)} \int_{0}^{\tau_{i}}\left(\tau_{i}-s\right)^{\alpha-1} f\left(s, x_{n}(s)\right) d s\right. \\
\left.\quad+\frac{1}{p(t) \Gamma(\alpha)} \int_{0}^{t}(t-s)^{\alpha-1} f\left(s, x_{n}(s)\right) d s\right\} \\
=\left\{\frac{K t^{\alpha-1}}{p(t)} \sum_{i=1}^{m} a_{i} \frac{\lim _{n \rightarrow \infty} g\left(\tau_{i}, x_{n}\left(\tau_{i}\right)\right.}{p\left(\tau_{i}\right) \Gamma(\alpha)} \int_{0}^{\tau_{i}}\left(\tau_{i}-s\right)^{\alpha-1} \lim _{n \rightarrow \infty} f\left(s, x_{n}(s)\right) d s\right. \\
\left.\quad+\frac{1}{p(t) \Gamma(\alpha)} \int_{0}^{t}(t-s)^{\alpha-1} \lim _{n \rightarrow \infty} f\left(s, x_{n}(s)\right) d s\right\} \\
\frac{K t^{\alpha-1}}{p(t)} \sum_{i=1}^{m} a_{i} \frac{g\left(\tau_{i}, x\left(\tau_{i}\right)\right.}{p\left(\tau_{i}\right) \Gamma(\alpha)} \int_{0}^{\tau_{i}}\left(\tau_{i}-s\right)^{\alpha-1} f(s, x(s)) d s \\
\left.+\frac{1}{p(t) \Gamma(\alpha)} \int_{0}^{t}(t-s)^{\alpha-1} f(s, x(s)) d s\right\}=\mathcal{B} x(t) .
\end{gathered}
$$

for all $t \in J$. This shows that $\mathcal{B} x_{n}$ converges monotonically to $\mathcal{B} x$ pointwise on $J$. 
Next, we will show that $\left\{\mathcal{B} x_{n}\right\}_{n \in \mathbb{N}}$ is an equicontinuous sequence of functions in $E$. Let $t_{1}, t_{2} \in J$ with $t_{1}<t_{2}$. Then

$$
\begin{aligned}
& \left|B x_{n}\left(t_{2}\right)-B x_{n}\left(t_{1}\right)\right| \\
& =\mid \frac{K t_{2}{ }^{\alpha-1}}{p\left(t_{2}\right)} \sum_{i=1}^{m} a_{i} \frac{g\left(\tau_{2_{i}}, x_{n}\left(\tau_{2_{i}}\right)\right)}{p\left(\tau_{2_{i}}\right) \Gamma(\alpha)} \int_{0}^{\tau_{2_{i}}}\left(\tau_{2_{i}}-s\right)^{\alpha-1} f\left(s, x_{n}(s)\right) d s \\
& +\frac{1}{p\left(t_{2}\right) \Gamma(\alpha)} \int_{0}^{t_{2}}\left(t_{2}-s\right)^{\alpha-1} f\left(s, x_{n}(s)\right) d s \\
& -\frac{K t_{1}{ }^{\alpha-1}}{p\left(t_{1}\right)} \sum_{i=1}^{m} a_{i} \frac{g\left(\tau_{1_{i}}, x_{n}\left(\tau_{1_{i}}\right)\right)}{p\left(\tau_{1_{i}}\right) \Gamma(\alpha)} \int_{0}^{\tau_{1_{i}}}\left(\tau_{1_{i}}-s\right)^{\alpha-1} f\left(s, x_{n}(s)\right) d s \\
& -\frac{1}{p\left(t_{1}\right) \Gamma(\alpha)} \int_{0}^{t_{1}}\left(t_{1}-s\right)^{\alpha-1} f\left(s, x_{n}(s)\right) d s \\
& =\mid \frac{K t_{2}{ }^{\alpha-1}}{p\left(t_{2}\right)} \sum_{i=1}^{m} a_{i} \frac{g\left(\tau_{2_{i}}, x_{n}\left(\tau_{2_{i}}\right)\right)}{p\left(\tau_{2_{i}}\right) \Gamma(\alpha)} \int_{0}^{\tau_{2_{i}}}\left(\tau_{2_{i}}-s\right)^{\alpha-1} f\left(s, x_{n}(s)\right) d s \\
& -\frac{K t_{1}^{\alpha-1}}{p\left(t_{1}\right)} \sum_{i=1}^{m} a_{i} \frac{g\left(\tau_{1_{i}}, x_{n}\left(\tau_{1_{i}}\right)\right)}{p\left(\tau_{1_{i}}\right) \Gamma(\alpha)} \int_{0}^{\tau_{1_{i}}}\left(\tau_{1_{i}}-s\right)^{\alpha-1} f\left(s, x_{n}(s)\right) d s \\
& +\left|\frac{1}{p\left(t_{2}\right) \Gamma(\alpha)} \int_{0}^{t_{2}}\left(t_{2}-s\right)^{\alpha-1} f\left(s, x_{n}(s)\right) d s-\frac{1}{p\left(t_{1}\right) \Gamma(\alpha)} \int_{0}^{t_{1}}\left(t_{1}-s\right)^{\alpha-1} f\left(s, x_{n}(s)\right) d s\right| \\
& \leq \mid \frac{K t_{2}{ }^{\alpha-1}}{p\left(t_{2}\right)} \sum_{i=1}^{m} a_{i} \frac{g\left(\tau_{2_{i}}, x_{n}\left(\tau_{2_{i}}\right)\right)}{p\left(\tau_{2_{i}}\right) \Gamma(\alpha)} \int_{0}^{\tau_{2_{i}}}\left(\tau_{2_{i}}-s\right)^{\alpha-1} f\left(s, x_{n}(s)\right) d s \\
& -\frac{K t_{2}^{\alpha-1}}{p\left(t_{2}\right)} \sum_{i=1}^{m} a_{i} \frac{g\left(\tau_{2_{i}}, x_{n}\left(\tau_{2_{i}}\right)\right)}{p\left(\tau_{2_{i}}\right) \Gamma(\alpha)} \int_{0}^{\tau_{2_{i}}}\left(\tau_{1_{i}}-s\right)^{\alpha-1} f\left(s, x_{n}(s)\right) d s \\
& +\mid \frac{K t_{2}{ }^{\alpha-1}}{p\left(t_{2}\right)} \sum_{i=1}^{m} a_{i} \frac{g\left(\tau_{2_{i}}, x_{n}\left(\tau_{2_{i}}\right)\right)}{p\left(\tau_{2_{i}}\right) \Gamma(\alpha)} \int_{0}^{\tau_{2_{i}}}\left(\tau_{1_{i}}-s\right)^{\alpha-1} f\left(s, x_{n}(s)\right) d s \\
& -\frac{K t_{1}{ }^{\alpha-1}}{p\left(t_{1}\right)} \sum_{i=1}^{m} a_{i} \frac{g\left(\tau_{1_{i}}, x_{n}\left(\tau_{1_{i}}\right)\right)}{p\left(\tau_{1_{i}}\right) \Gamma(\alpha)} \int_{0}^{\tau_{1_{i}}}\left(\tau_{1_{i}}-s\right)^{\alpha-1} f\left(s, x_{n}(s)\right) d s
\end{aligned}
$$




$$
\begin{aligned}
& +\left|\frac{1}{p\left(t_{2}\right) \Gamma(\alpha)} \int_{0}^{t_{2}}\left(t_{2}-s\right)^{\alpha-1} f\left(s, x_{n}(s)\right) d s-\frac{1}{p\left(t_{2}\right) \Gamma(\alpha)} \int_{0}^{t_{2}}\left(t_{1}-s\right)^{\alpha-1} f\left(s, x_{n}(s)\right) d s\right| \\
& +\left|\frac{1}{p\left(t_{2}\right) \Gamma(\alpha)} \int_{0}^{t_{2}}\left(t_{1}-s\right)^{\alpha-1} f\left(s, x_{n}(s)\right) d s-\frac{1}{p\left(t_{1}\right) \Gamma(\alpha)} \int_{0}^{t_{1}}\left(t_{1}-s\right)^{\alpha-1} f\left(s, x_{n}(s)\right) d s\right| \\
& \leq\left|\frac{K t_{2}{ }^{\alpha-1}}{p\left(t_{2}\right)} \sum_{i=1}^{m} a_{i} \frac{g\left(\tau_{2_{i}}, x_{n}\left(\tau_{2_{i}}\right)\right)}{p\left(\tau_{2_{i}}\right) \Gamma(\alpha)}\right| \int_{0}^{\tau_{2_{i}}}\left|\left(\tau_{2_{i}}-s\right)^{\alpha-1}-\left(\tau_{1_{i}}-s\right)^{\alpha-1}\right| f\left(s, x_{n}(s)\right) d s \\
& +\left|\frac{K t_{2}{ }^{\alpha-1}}{p\left(t_{2}\right)} \sum_{i=1}^{m} a_{i} \frac{g\left(\tau_{2_{i}}, x_{n}\left(\tau_{2_{i}}\right)\right)}{p\left(\tau_{2_{i}}\right) \Gamma(\alpha)}-\frac{K t_{1}{ }^{\alpha-1}}{p\left(t_{1}\right)} \sum_{i=1}^{m} a_{i} \frac{g\left(\tau_{1_{i}}, x_{n}\left(\tau_{1_{i}}\right)\right)}{p\left(\tau_{1_{i}}\right) \Gamma(\alpha)}\right|\left|\int_{\tau_{1}}^{\tau_{2}}\left(\tau_{1_{i}}-s\right)^{\alpha-1} f\left(s, x_{n}(s)\right) d s\right| \\
& +\left|\frac{1}{p\left(t_{2}\right) \Gamma(\alpha)}\right| \int_{0}^{t_{2}}\left|\left(t_{2}-s\right)^{\alpha-1}-\left(t_{1}-s\right)^{\alpha-1}\right| f\left(s, x_{n}(s)\right) d s \\
& +\left|\frac{1}{p\left(t_{2}\right) \Gamma(\alpha)}-\frac{1}{p\left(t_{1}\right) \Gamma(\alpha)}\right|\left|\int_{t_{1}}^{t_{2}}\left(t_{1}-s\right)^{\alpha-1} f\left(s, x_{n}(s)\right) d s\right| \\
& \leq\left|\frac{K M}{p\left(t_{2}\right)} \sum_{i=1}^{m} a_{i} \frac{M_{g}}{p\left(\tau_{2_{i}}\right) \Gamma(\alpha)}\right| \int_{0}^{T}\left|\left(\tau_{2_{i}}-s\right)^{\alpha-1}-\left(\tau_{1_{i}}-s\right)^{\alpha-1}\right| M_{f} d s \\
& +\left|\frac{K M}{p\left(t_{2}\right)} \sum_{i=1}^{m} a_{i} \frac{M_{g}}{p\left(\tau_{2_{i}}\right) \Gamma(\alpha)}-\frac{K M}{p\left(t_{1}\right)} \sum_{i=1}^{m} a_{i} \frac{M_{g}}{p\left(\tau_{1_{i}}\right) \Gamma(\alpha)}\right|\left|t_{2}-t_{1}\right| M_{f} \\
& +\left|\frac{1}{p\left(t_{2}\right) \Gamma(\alpha)}\right| \int_{0}^{T}\left|\left(t_{2}-s\right)^{\alpha-1}-\left(t_{1}-s\right)^{\alpha-1}\right| M_{f} d s \\
& +\left|\frac{1}{p\left(t_{2}\right) \Gamma(\alpha)}-\frac{1}{p\left(t_{1}\right) \Gamma(\alpha)}\right|\left|t_{2}-t_{1}\right| M_{f} .
\end{aligned}
$$

Since the functions $\alpha$ is continuous on compact interval $J$ so uniformly continuous there. Therefore, from the above inequality (15) it follows that

$$
\left|\mathcal{B} x_{n}\left(t_{2}\right)-\mathcal{B} x_{n}\left(t_{1}\right)\right| \rightarrow 0 \quad \text { as } \quad n \rightarrow \infty
$$

uniformly for all $n \in \mathbb{N}$. This shows that the convergence $\mathcal{B} x_{n} \rightarrow \mathcal{B} x$ is uniform and hence $\mathcal{B}$ is partially continuous on $E$. 
Step IV: $\mathcal{B}$ is uniformly partially compact operator on $E$.

Let $C$ be an arbitrary chain in $E$. We show that $\mathcal{B}(C)$ is a uniformly bounded and equicontinuous set in $E$. First we show that $\mathcal{B}(C)$ is uniformly bounded. Let $y \in \mathcal{B}(C)$ be any element. Then there is an element $x \in C$ be such that $y=\mathcal{B} x$. Now, by hypothesis,

$$
\begin{aligned}
|y(t)| & =\mid \frac{K t_{2}{ }^{\alpha-1}}{p\left(t_{2}\right)} \sum_{i=1}^{m} a_{i} \frac{g\left(\tau_{2_{i}}, x_{n}\left(\tau_{2_{i}}\right)\right)}{p\left(\tau_{2_{i}}\right) \Gamma(\alpha)} \int_{0}^{\tau_{2_{i}}}\left(\tau_{2_{i}}-s\right)^{\alpha-1} f\left(s, x_{n}(s)\right) d s \\
& +\frac{1}{p\left(t_{2}\right) \Gamma(\alpha)} \int_{0}^{t_{2}}\left(t_{2}-s\right)^{\alpha-1} f\left(s, x_{n}(s)\right) d s \mid \leq \frac{K M\left[M_{G}+1\right] T^{\alpha-1} M_{f}}{p(T) \Gamma(\alpha)}=r
\end{aligned}
$$

for all $t \in J$. Taking the supremum over $t$, we obtain $\|y\| \leq\|\mathcal{B} x\| \leq r$ for all $y \in \mathcal{B}(C)$. Hence, $\mathcal{B}(C)$ is a uniformly bounded subset of $E$. Moreover, $\|\mathcal{B}(C)\| \leq r$ for all chains $C$ in $E$. Hence, $\mathcal{B}$ is a uniformly partially bounded operator on $E$.

Next, we will show that $\mathcal{B}(C)$ is an equicontinuous set in $E$. Let $t_{1}, t_{2} \in J$ with $t_{1}<t_{2}$. Then, for any $y \in \mathcal{B}(C)$, one has

$$
\begin{aligned}
\mid B x\left(t_{2}\right)- & B x\left(t_{1}\right) \mid \\
= & \mid \frac{K t_{2}{ }^{\alpha-1}}{p\left(t_{2}\right)} \sum_{i=1}^{m} a_{i} \frac{g\left(\tau_{2_{i}}, x\left(\tau_{2_{i}}\right)\right)}{p\left(\tau_{2_{i}}\right) \Gamma(\alpha)} \int_{0}^{\tau_{2_{i}}}\left(\tau_{2_{i}}-s\right)^{\alpha-1} f(s, x(s)) d s \\
& +\frac{1}{p\left(t_{2}\right) \Gamma(\alpha)} \int_{0}^{t_{2}}\left(t_{2}-s\right)^{\alpha-1} f(s, x(s)) d s \\
& -\frac{K t_{1}{ }^{\alpha-1}}{p\left(t_{1}\right)} \sum_{i=1}^{m} a_{i} \frac{g\left(\tau_{1_{i}}, x\left(\tau_{1_{i}}\right)\right)}{p\left(\tau_{1_{i}}\right) \Gamma(\alpha)} \int_{0}^{\tau_{1_{i}}}\left(\tau_{1_{i}}-s\right)^{\alpha-1} f(s, x(s)) d s \\
& -\frac{1}{p\left(t_{1}\right) \Gamma(\alpha)} \int_{0}^{t_{1}}\left(t_{1}-s\right)^{\alpha-1} f(s, x(s)) d s \mid \\
= & \quad \frac{K t_{2}{ }^{\alpha-1}}{p\left(t_{2}\right)} \sum_{i=1}^{m} a_{i} \frac{g\left(\tau_{2_{i}}, x\left(\tau_{2_{i}}\right)\right)}{p\left(\tau_{2_{i}}\right) \Gamma(\alpha)} \int_{0}^{\tau_{2_{i}}}\left(\tau_{2_{i}}-s\right)^{\alpha-1} f(s, x(s)) d s \\
& \frac{K t_{1}{ }^{\alpha-1}}{p\left(t_{1}\right)} \sum_{i=1}^{m} a_{i} \frac{g\left(\tau_{1_{i}}, x\left(\tau_{1_{i}}\right)\right)}{p\left(\tau_{1_{i}}\right) \Gamma(\alpha)} \int_{0}^{\tau_{1_{i}}}\left(\tau_{1_{i}}-s\right)^{\alpha-1} f(s, x(s)) d s \mid
\end{aligned}
$$




$$
\begin{aligned}
& +\left|\frac{1}{p\left(t_{2}\right) \Gamma(\alpha)} \int_{0}^{t_{2}}\left(t_{2}-s\right)^{\alpha-1} f(s, x(s)) d s-\frac{1}{p\left(t_{1}\right) \Gamma(\alpha)} \int_{0}^{t_{1}}\left(t_{1}-s\right)^{\alpha-1} f(s, x(s)) d s\right| \\
& \leq \mid \frac{K t_{2}{ }^{\alpha-1}}{p\left(t_{2}\right)} \sum_{i=1}^{m} a_{i} \frac{g\left(\tau_{2_{i}}, x\left(\tau_{2_{i}}\right)\right)}{p\left(\tau_{2_{i}}\right) \Gamma(\alpha)} \int_{0}^{\tau_{2_{i}}}\left(\tau_{2_{i}}-s\right)^{\alpha-1} f(s, x(s)) d s \\
& -\frac{K t_{2}{ }^{\alpha-1}}{p\left(t_{2}\right)} \sum_{i=1}^{m} a_{i} \frac{g\left(\tau_{2_{i}}, x\left(\tau_{2_{i}}\right)\right)}{p\left(\tau_{2_{i}}\right) \Gamma(\alpha)} \int_{0}^{\tau_{2_{i}}}\left(\tau_{1_{i}}-s\right)^{\alpha-1} f(s, x(s)) d s \\
& +\mid \frac{K t_{2}{ }^{\alpha-1}}{p\left(t_{2}\right)} \sum_{i=1}^{m} a_{i} \frac{g\left(\tau_{2_{i}}, x\left(\tau_{2_{i}}\right)\right)}{p\left(\tau_{2_{i}}\right) \Gamma(\alpha)} \int_{0}^{\tau_{2_{i}}}\left(\tau_{1_{i}}-s\right)^{\alpha-1} f(s, x(s)) d s \\
& -\frac{K t_{1}{ }^{\alpha-1}}{p\left(t_{1}\right)} \sum_{i=1}^{m} a_{i} \frac{g\left(\tau_{1_{i}}, x\left(\tau_{1_{i}}\right)\right)}{p\left(\tau_{1_{i}}\right) \Gamma(\alpha)} \int_{0}^{\tau_{1_{i}}}\left(\tau_{1_{i}}-s\right)^{\alpha-1} f(s, x(s)) d s \\
& +\left|\frac{1}{p\left(t_{2}\right) \Gamma(\alpha)} \int_{0}^{t_{2}}\left(t_{2}-s\right)^{\alpha-1} f(s, x(s)) d s-\frac{1}{p\left(t_{2}\right) \Gamma(\alpha)} \int_{0}^{t_{2}}\left(t_{1}-s\right)^{\alpha-1} f(s, x(s)) d s\right| \\
& +\left|\frac{1}{p\left(t_{2}\right) \Gamma(\alpha)} \int_{0}^{t_{2}}\left(t_{1}-s\right)^{\alpha-1} f(s, x(s)) d s-\frac{1}{p\left(t_{1}\right) \Gamma(\alpha)} \int_{0}^{t_{1}}\left(t_{1}-s\right)^{\alpha-1} f(s, x(s)) d s\right| \\
& \leq\left|\frac{K t_{2}{ }^{\alpha-1}}{p\left(t_{2}\right)} \sum_{i=1}^{m} a_{i} \frac{g\left(\tau_{2_{i}}, x\left(\tau_{2_{i}}\right)\right)}{p\left(\tau_{2_{i}}\right) \Gamma(\alpha)}\right| \int_{0}^{\tau_{2_{i}}}\left|\left(\tau_{2_{i}}-s\right)^{\alpha-1}-\left(\tau_{1_{i}}-s\right)^{\alpha-1}\right| f(s, x(s)) d s \\
& +\left|\frac{K t_{2}{ }^{\alpha-1}}{p\left(t_{2}\right)} \sum_{i=1}^{m} a_{i} \frac{g\left(\tau_{2_{i}}, x\left(\tau_{2_{i}}\right)\right)}{p\left(\tau_{2_{i}}\right) \Gamma(\alpha)}-\frac{K t_{1}{ }^{\alpha-1}}{p\left(t_{1}\right)} \sum_{i=0}^{m} a_{i} \frac{g\left(\tau_{1_{i}}, x\left(\tau_{1_{i}}\right)\right)}{p\left(\tau_{1_{i}}\right) \Gamma(\alpha)}\right|\left|\int_{\tau_{1}}^{\tau_{2}}\left(\tau_{1_{i}}-s\right)^{\alpha-1} f(s, x(s)) d s\right| \\
& +\left|\frac{1}{p\left(t_{2}\right) \Gamma(\alpha)}\right| \int_{0}^{t_{2}}\left|\left(t_{2}-s\right)^{\alpha-1}-\left(t_{1}-s\right)^{\alpha-1}\right| f(s, x(s)) d s \\
& +\left|\frac{1}{p\left(t_{2}\right) \Gamma(\alpha)}-\frac{1}{p\left(t_{1}\right) \Gamma(\alpha)}\right|\left|\int_{t_{1}}^{t_{2}}\left(t_{1}-s\right)^{\alpha-1} f(s, x(s)) d s\right| \\
& \leq\left|\frac{K M}{p\left(t_{2}\right)} \sum_{i=1}^{m} a_{i} \frac{M_{g}}{p\left(\tau_{2_{i}}\right) \Gamma(\alpha)}\right| \int_{0}^{T}\left|\left(\tau_{2_{i}}-s\right)^{\alpha-1}-\left(\tau_{1_{i}}-s\right)^{\alpha-1}\right| M_{f} d s
\end{aligned}
$$




$$
\begin{aligned}
& \leq\left|\frac{K M}{p\left(t_{2}\right)} \sum_{i=1}^{m} a_{i} \frac{M_{g}}{p\left(\tau_{2_{i}}\right) \Gamma(\alpha)}\right| \int_{0}^{T}\left|\left(\tau_{2_{i}}-s\right)^{\alpha-1}-\left(\tau_{1_{i}}-s\right)^{\alpha-1}\right| M_{f} d s \\
& +\left|\frac{K M}{p\left(t_{2}\right)} \sum_{i=1}^{m} a_{i} \frac{M_{g}}{p\left(\tau_{2_{i}}\right) \Gamma(\alpha)}-\frac{K M}{p\left(t_{1}\right)} \sum_{i=1}^{m} a_{i} \frac{M_{g}}{p\left(\tau_{1_{i}}\right) \Gamma(\alpha)}\right|\left|t_{2}-t_{1}\right| M_{f} \\
& +\left|\frac{1}{p\left(t_{2}\right) \Gamma(\alpha)}\right| \int_{0}^{T}\left|\left(t_{2}-s\right)^{\alpha-1}-\left(t_{1}-s\right)^{\alpha-1}\right| M_{f} d s \\
& +\left|\frac{1}{p\left(t_{2}\right) \Gamma(\alpha)}-\frac{1}{p\left(t_{1}\right) \Gamma(\alpha)}\right|\left|t_{2}-t_{1}\right| M_{f} \\
& \longrightarrow 0 \quad \text { as } t_{1} \rightarrow t_{2},
\end{aligned}
$$

uniformly for all $y \in \mathcal{B}(C)$. Hence $\mathcal{B}(C)$ is an equicontinuous subset of $E$. Now, $\mathcal{B}(C)$ is a uniformly bounded and equicontinuous set of functions in $E$, so it is compact. Consequently, $\mathcal{B}$ is a uniformly partially compact operator on $E$ into itself.

Step V: $u_{l}$ satisfies the operator inequality $u_{l} \leq \mathcal{A} u_{l} \mathcal{B} u_{l}$.

By hypothesis $\left(\mathrm{A}_{5}\right)$, the FDE 5 has a lower solution $u_{l}$ defined on $J$. Then, we have

$$
\begin{aligned}
u_{l}(t) & \leq g\left(t, u_{l}(t)\right)\left\{\frac{K t_{2}{ }^{\alpha-1}}{p\left(t_{2}\right)} \sum_{i=1}^{m} a_{i} \frac{g\left(\tau_{2_{i}}, u_{l}\left(\tau_{2_{i}}\right)\right)}{p\left(\tau_{2_{i}}\right) \Gamma(\alpha)} \int_{0}^{\tau_{2_{i}}}\left(\tau_{2_{i}}-s\right)^{\alpha-1} f\left(s, u_{l}(s)\right) d s\right. \\
& \left.+\frac{1}{p\left(t_{2}\right) \Gamma(\alpha)} \int_{0}^{t_{2}}\left(t_{2}-s\right)^{\alpha-1} f\left(s, u_{l}(s)\right) d s\right\},
\end{aligned}
$$

for all $t \in J$. From the definitions of the operators $\mathcal{A}, \mathcal{B}$ and $\mathcal{C}$ it follows that $u_{l}(t) \leq \mathcal{A} u_{l}(t) \mathcal{B} u_{l}(t)$ for all $t \in J$. Hence $u_{l} \leq \mathcal{A} u_{l} \mathcal{B} u_{l}$.

Step VI: The D-functions $\psi_{\mathcal{A}}$ satisfy the growth condition $M \psi_{\mathcal{A}}(r)<r$, for $r>0$.

Finally, the $\mathcal{D}$-function $\psi_{\mathcal{A}}$ of the operator $\mathcal{A}$ satisfy the inequality given in hypothesis of Theorem 9, viz.,

$$
M \psi_{\mathcal{A}}(r) \leq\left(\frac{K M\left[M_{G}+1\right] T^{\alpha-1} M_{f}}{p(T) \Gamma(\alpha)}\right) \psi_{f}(r)<r
$$

for all $r>0$.

Thus $\mathcal{A}$ and $\mathcal{B}$ satisfy all the conditions of Theorem 9 and we conclude that the operator equation $\mathcal{A} x \mathcal{B} x=x$ has a solution. Consequently the FDE (5) has a solution $x^{*}$ defined on $J$. Furthermore, the sequence $\left\{x_{n}\right\}_{n \in \mathbb{N}}$ of successive approximations defined by (13) converges monotonically to $x^{*}$. This completes the proof.

The conclusion of Theorems 14 also remains true if we replace the hypothesis $\left(\mathrm{A}_{5}\right)$ with the following one:

$\left(\mathrm{A}_{5}^{\prime}\right)$ The FDE (5) has an upper solution $u_{u} \in C(J, \mathbb{R})$.

The proof of Theorem 14 under this new hypothesis is similar and can be obtained by closely observing the same arguments with appropriate modifications. 


\section{References}

[1] P.L. Butzer, A.A. Kilbas, J.J. Trujillo, Fractional calculus in the Mellin setting and Hadamard-type fractional integrals, Journal of Mathematical Analysis and Applications. 269(1) (2002) 1-27.

[2] P.L. Butzer, A.A. Kilbas, J.J. Trujillo, Mellin transform analysis and integration by parts for Hadamard-type fractional integrals, Journal of Mathematical Analysis and Applications. 270(1) (2002) 1-15.

[3] B.C. Dhage, Partially condensing mappings in ordered normed linear spaces and applications to functional integral equations, Tamkang J. Math. 45(4) (2014) 397-426.

[4] B.C. Dhage, Nonlinear $\mathcal{D}$-set-contraction mappings in partially ordered normed linear spaces and applications to functional hybrid integral equations, Malaya J. Mat. 3(1) (2015) 62-85.

[5] B.C. Dhage, Operator theoretic techniques in the theory of nonlinear hybrid differential equations, Nonlinear Anal. Forum. 20 (2015) 15-31.

[6] A.M.A. El-Sayed, H.H.G. Hashem, Integrable and continuous solutions of a nonlinear quadratic integral equation, Electronic Journal of Qualitative Theory of Differential Equations. 25 (2008) $1-10$.

[7] A.M.A. El-Sayed, H.H.G. Hashem, Existence results for nonlinear quadratic integral equations of fractional order in Banach algebra, Fract. Calc. Appl. Anal. 16(4) (2013) 816-826.

[8] S. Heikkilä, V. Lakshmikantham, Monotone iterative techniques for discontinuous nonlinear differential equations, Marcel Dekker inc., New York, 1994.

[9] V. Lakshmikantham, S. Leela, Differential and integral inequalities, Vol. I, New York, London, 1909.

[10] A.A. Kilbas, H.M. Srivastava, J.J. Trujillo, Theory and applications of fractional differential equations, North-Holland Mathematics Studies, 204, Elsevier Science B.V., Amsterdam, 2006.

[11] K.S. Miller, B. Ross, An introduction to the fractional calculus and fractional differential equations, John Wiley, New York, 1993.

[12] I. Podlubny, Fractional differential equations, Academic Press, San Diego, 1999.

[13] K.M. Furati, N.E. Tatar, Power-type estimates for nonlinear fractional differential equation, Nonlinear Anal. 62(6) (2005) 1025-1036.

[14] Z. Weia, W. Dongc, J. Chea, Periodic boundary value problems for fractional differential equations involving a Riemann-Liouville fractional derivative, Nonlinear Analysis: Theory, Methods \& Applications. 73(10) (2010) 3232-3238.

[15] T. Jankowski, Fractional equations of Volterra type involving a Riemann-Liouville derivative, Appl. Math. Lett. 26(3) (2013) 344-350.

[16] F.M. Gaaffar, Continuous and integrable solutions of a nonlinear Cauchy problem of fractional order with nonlocal conditions, Journal of the Egyptian Mathematical Society. 22(3) (2014) 341347.

[17] I.K. Argyros, Quadratic equations and applications to Chandrasekhar's and related equations, Bull. Austral. Math. Soc. 32(2) (1985) 275-292. 
[18] S. Chandrasekhar, Radiative transfer, Dover Publications, New York, 1960.

[19] M.A. Darwish, S.K. Ntouyas, Monotonic solutions of a perturbed quadratic fractional integral equation, Nonlinear Analysis: Theory, Methods \& Applications. 71(11) (2009) 5513-5521.

[20] K. Deimling, Nonlinear fuctional analysis, Springer-Verlag, Berlin, 1985.

[21] B.C. Dhage, A nonlinear alternative in Banach algebras with applications to functional differential equations, Nonlinear Funct. Anal. \& Appl. 8 (2004) 563-575.

[22] B.C. Dhage, Fixed point theorems in ordered Banach algebras and applications, PanAmer. Math. J. 9(4) (1999) 93-102.

[23] B.R. Sontakke, G.P. Kamble, M. Mazhar-ul-Haque, Some integral transform of generalized Mittag-Lefller functions, International Journal of Pure and Applied Mathematics. 108(2) (2016) $327-339$.

[24] T.L. Holambe, M. Mazhar-ul-Haque, A remark on semigroup property in fractional calculus, International Journal of Mathematics and Computer Application Research. 4(6) (2014) 27-32.

[25] M. Mazhar-ul-Haque, T.L. Holambe, A Q function in fractional calculus, Journal of Basic and Applied Research International. 6(4) (2015) 248-252.

[26] T.L. Holambe, M. Mazhar - Ul-Haque, G.P. Kamble, Approximations to the solution of Cauchy type weighted nonlocal fractional differential equation, Nonlinear Analysis and Differential Equations. 4(15) (2016) 697-717.

[27] M. Mazhar-ul-Haque, T.L. Holambe, G.P. Kamble, Solution to weighted non-local fractional differential equation, International Journal of Pure and Applied Mathematics. 108(1) (2016) 79 91.

[28] M. Mazhar-ul-Haque, T.L. Holambe, Positive solutions of quadratic fractional integral equation with generalized Mittag-Leffler function, International Journal of Mathematics and its Applications. 5(1-A) (2017) 65-74. 